\title{
Real-time Monitoring of Macromolecular Biosensing Probe Self- assembly and On-chip ELISA using Impedimetric Microsensors
}

\author{
Faheng Zang ${ }^{\text {a }}$, Konstantinos Gerasopoulos a , Xiao Zhu Fan ${ }^{\text {a }, ~ A d a m ~ D . ~ B r o w n ~}{ }^{\text {b }}$, \\ James N. Culver ${ }^{\mathrm{c}}$, and Reza Ghodssi ${ }^{\text {a, }}$ \\ University of Maryland, College Park, Maryland, USA

\section{Author and Co-author Contact Information} \\ ${ }^{a}$ Department of Electrical and Computer Engineering, Institute for Systems Research \\ ${ }^{\mathrm{b}}$ Fischell Department of Bioengineering \\ ${ }^{c}$ Institute for Bioscience and Biotechnology Research and Department of Plant Science and \\ Landscape Architecture \\ University of Maryland, College Park, USA \\ *Corresponding author:
}

Fax: +1 301314 9920; Tel: +1 301405 8158; E-mail: ghodssi@umd.edu (Reza Ghodssi)

\begin{abstract}
This paper presents a comprehensive study of the self-assembly dynamics and the biosensing efficacy of Tobacco mosaic virus-like particle (TMV VLP) sensing probes using an impedimetric microsensor platform. TMV VLPs are high surface area macromolecules with nanorod structures constructed from helical arrangements of thousands of identical coat proteins. Genetically modified VLPs express both surface attachment-promoting cysteine residues and FLAG-tag antibody binding peptides on their coat protein outer surfaces, making them selective biosensing probes with self-assembly capability on sensors. The VLP self-assembly dynamics were studied by the continuous monitoring of impedance changes at $100 \mathrm{~Hz}$ using interdigitated impedimetric microsensors. Electrical impedance spectroscopy revealed VLP saturation on impedance sensor surface in 8 hours with surface coverage of $68 \%$ in selfassembly process. The VLP-functionalized impedance sensors responded to $12 \mathrm{ng} / \mathrm{ml}-1.2 \mu \mathrm{g} / \mathrm{ml}$ of target anti-FLAG IgG antibodies in the subsequent enzyme-linked immunosorbent assays (ELISA), and yielded 18\% - 35\% total impedance increases, respectively. The detection limit of the target antibody is $9.1 \mathrm{ng} / \mathrm{ml}$ using the VLP-based impedimetric microsensor. These results highlight the significant potential of genetically modified VLPs as selective nanostructured probes for autonomous sensor functionalization and enhanced biosensing.
\end{abstract}

\section{Keywords}

Virus-like particle, impedance sensor, microfabrication, macromolecule, bioreceptor

\section{Introduction}

Selective and sensitive detection of pathogens are crucial steps in identifying sources of disease outbreaks and forming effective strategies to ensure public health and food safety (Mandal et al. 2011; Yang and Bashir 2008). Among the current most effective and widely used biosensing methods, immunoassays, such as enzyme-linked immunosorbent assays (ELISA), are implemented to sense specific types of pathogens based on receptor-target interactions. Researchers have integrated the conventional lab-scale immunoassays with microfabrication technologies, and developed micro biosensors based on signal changes from bioluminescence, impedance or piezoelectric responses during the target-receptor interactions (Babacan et al. 2000; Katz and Willner 2003; Narsaiah et al. 2012; Roda and Guardigli 2012; Roda et al. 2004). In these microfabricated biosensors, receptor 
molecules with high affinity to target molecules are used to functionalize the transducer surfaces to achieve high selectivity. The efficacy of sensitive and selective biosensing is therefore largely determined by the density and binding affinity of the functional receptor layer. Currently, antibodies are the most commonly used receptors due to their versatility, convenience in handling and selectivity to targets (Mandal et al. 2011). However, a major problem associated with antibody sensing probes in immunoassays is their low capture efficiency on the transducer surface (Varshney et al. 2007). Limited by this, surface functionalization is not maximized and the sensitivity of the immunoassay is not optimized. Moreover, most surface functionalization procedures for immunoassays require several steps of chemical treatments on the surface. This prolongs the immunoassay, making it a time consuming and complex process. For these reasons, there is a need to develop sensing probes that are capable of functionalizing sensor surfaces densely and rapidly.

Tobacco mosaic virus (TMV) and its derivative virus-like particle (VLP) are emerging biomaterials that demonstrate viability as biosensing probes (Culver et al. 2015; Fan et al. 2013). TMV, a cylindrical nanostructured filamentous plant virus with 2130 identical coat proteins exposed on its outer surface, can express programmable biorecognition affinities through genetic modifications to its coat proteins. These modifications include adding cysteines that facilitate self-assembly onto various substrates and peptides with high affinity to target molecules. Owing to its high aspect ratio, the vertically self-assembled TMV is able to exhibit thousands of binding receptors on its nanorod outer surface while maintaining a small footprint on a sensor surface. TMV applications in microdevices span from biological nano-scaffolds for surface decoration in energy storage devices to high affinity sensing probes for selectivity-enhanced bio- and chemical sensing (Fan et al. 2010; Gerasopoulos et al. 2012). The most recent research has successfully demonstrated the process of rapid and massive production of the functional TMV coat proteins inside bacteria (Brown et al. 2013). Though lacking the central RNA strand, these coat proteins self-assemble into nanorod-structured virus-like particles (VLPs) similar to TMVs in the absence of RNA strain, with a much higher yield. VLPs are capable of creating dense functional surfaces in sensors for selective sensing of the biological binding events.

Previously, VLPs were used both as free floating particles in solution to selectively sense the presence of TNT explosives and as immobilized receptors on optical disk resonators for antibody sensing (Zang et al. 2013; Zang et al. 2014; Fan et al. 2015). In the latter case, the VLP functionalized optical resonators were able to sense antibody attachment by measuring the endpoint resonant frequency shifts in air. The optical sensors required the biological samples to be dried in order to achieve a high sensitivity in the endpoint measurement. Thus, the drying and rewetting processes were the bottlenecks that limited device stability and the potential to perform a real-time monitoring. Consequently, an improved sensing platform needs to be developed to monitor the sensing probe and target molecule interactions in liquid environments. As a promising candidate to solve these challenges, electrical impedance spectroscopy has become a rapidly developing technique with the capability to directly probe biological binding-induced capacitance and resistance changes on an electrode or substrate surface. The technique also provides suitable platforms for continuous study of macromolecular binding in liquid (Katz and Willner 2003; Van Gerwen et al. 1998; Zou et al. 2007). Electrical impedance microsensors have been used to analyze ionic strength as well as detect DNA hybridization events or the presence of pathogenic bacteria in solution (Ayliffe et al. 1999; MorenoHagelsieb et al. 2004; Yang and Bashir 2008).

In this work, the capabilities of impedance microsensors such as good compatibility with liquid samples and high sensitivity to surface attachment events are leveraged to achieve direct and real-time monitoring of VLP sensing probe self-assembly and ELISA on-chip. VLPs expressing cysteine and 
FLAG-tag peptides (VLP-FLAG) functional groups are used as sensing probes showing high affinity to the target anti-FLAG antibody. The electrical impedance between the interdigitated microelectrodes (IDMEs) is continuously monitored in the VLP-FLAG self-assembly process to understand its dynamics. The biosensing efficacy is determined by performing ELISA utilizing the VLP-functionalized impedance microsensors. Both VLP-FLAG sensing probe concentration and the microelectrode feature size are studied to achieve sensitivity-enhanced VLP-based biosensors.

\section{Materials and Methodologies}

2.1 Tobacco mosaic virus-like particles: high receptor density biosensing probes

The VLP-FLAG biosensing probes are synthetized in Escherichia coli bacterial cell hosts by expression and subsequent helical auto-assembly of thousands of genetically modified, identical TMV coat proteins.

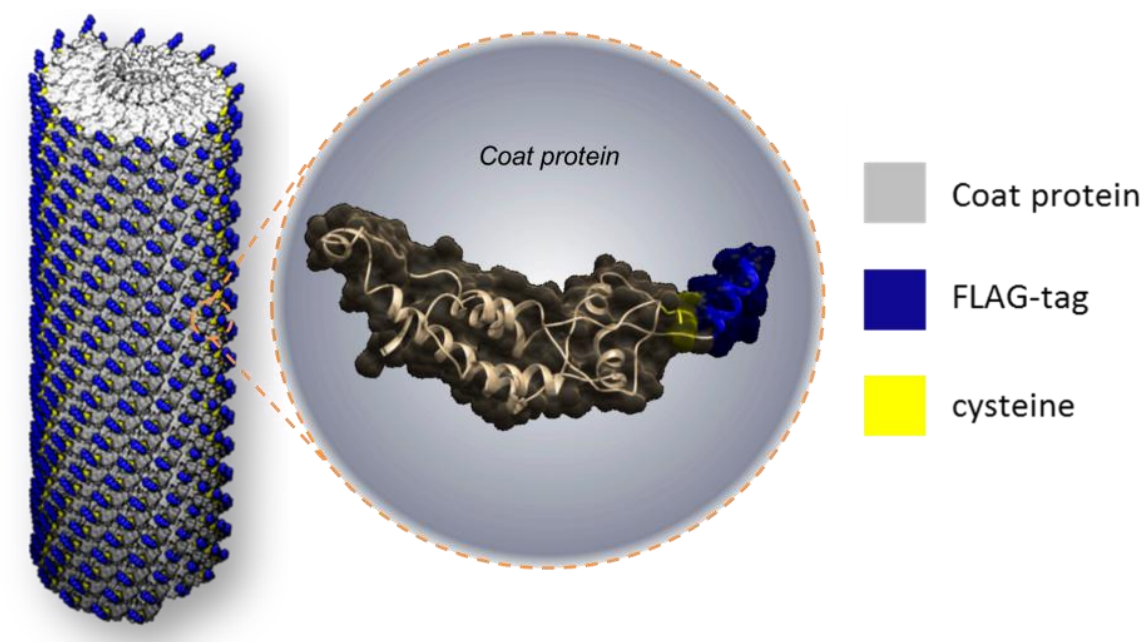

\section{Figure 1: Schematic of a three dimensional segment of VLP with helical arrangement of genetically modified cysteine residues and FLAG-tag sequences on coat proteins}

Figure 1 shows the three-dimensional segment of a VLP-FLAG sensing probe, a nanorod structure with $18 \mathrm{~nm}$ in outer diameter and up to micrometers in length. Each coat protein expresses a cysteine residue (yellow) that promotes surface attachment and a FLAG-tag sequence (DYKDDDDK; blue) that enables selective binding with the target anti-FLAG antibody. Due the location and size difference between the cysteines and peptides, the attachment - promoting cysteines are only exposed at the one end of a VLP nanorod. This enables the VLP to self-assemble on surfaces vertically, texturing a planar surface with high-surface-area functional nanostructures.

The genetic modification, culture and purification procedures were reported in detail previously (Brown et al. 2013). The maximum concentration of VLPs after purification can reach up to 0.6 $\mathrm{mg} / \mathrm{ml}$ in $0.1 \mathrm{M}$ sodium phosphate buffer solutions. The purified VLPs were diluted and suspended in $0.1 \mathrm{M}$ sodium phosphate buffer $(\mathrm{pH}=7$ ) at a previously characterized concentration of $0.2 \mathrm{mg} / \mathrm{ml}$ for effective surface functionalization. In the later experiments and discussions, impedance sensors functionalized by either VLP concentration $(0.6 \mathrm{mg} / \mathrm{ml}$ or $0.2 \mathrm{mg} / \mathrm{ml})$ will be compared in their biosensing efficacy in ELISA. All chemicals used in buffer solutions preparations are from Fisher Scientific. The solutions are based on deionized (DI) water (resistivity equals $18 \mathrm{M} \Omega \cdot \mathrm{cm}$ ) from EPure Ultrapure Water Purification Systems (Thermo Scientific).

\subsection{Design, fabrication and modelling of impedance microsensor}




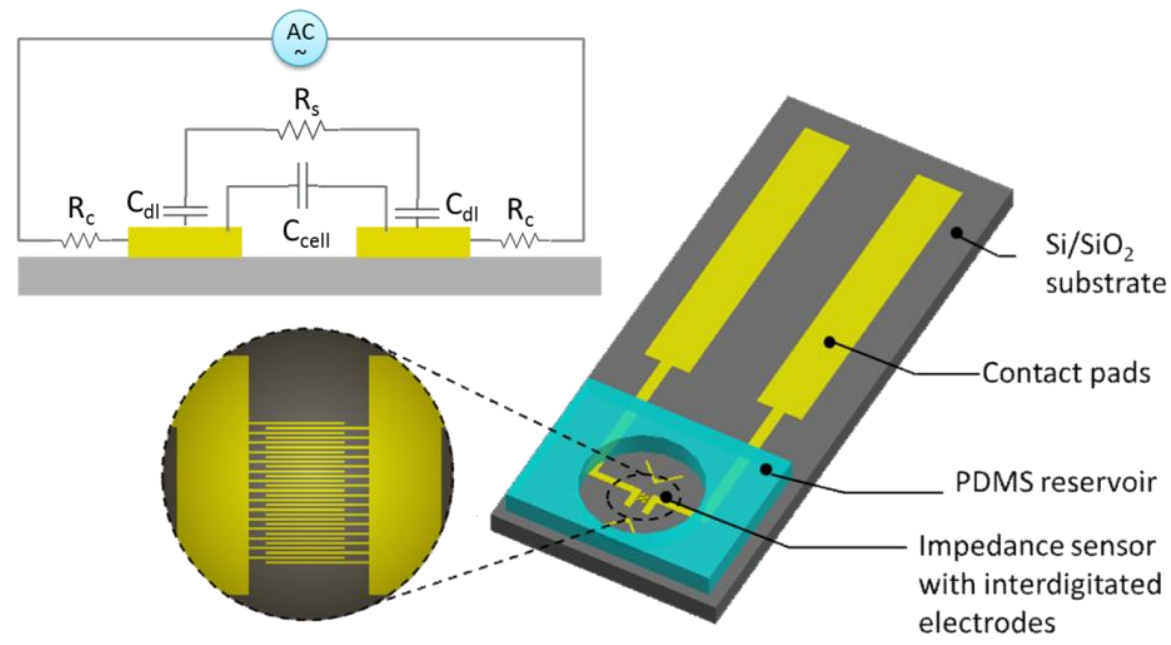

Figure 2. Schematic of the impedance microsensor

Figure 2 shows a schematic of the impedance microsensor which comprises gold IDMEs, contact pads and a PDMS reaction chamber. In the fabrication process, a $500 \mathrm{~nm}$ thick $\mathrm{SiO}_{2}$ layer was fabricated using plasma enhanced chemical vapour deposition (PECVD) (Plasmalab System 100, Oxford Instruments) on a $500 \mu \mathrm{m}$ thick silicon wafer. A $1.6 \mu \mathrm{m}$ thick layer of Shipley 1813 positive photoresist was spin-coated and patterned via photolithography to form contact pad and impedance sensor patterns. The exposed $\mathrm{SiO}_{2}$ area after the photolithography was isotropically wet-etched in buffered HF to form $200 \mathrm{~nm}$ undercuts under the photoresist. Cr/Au (200 $\mathrm{A} / 1400 \AA$ ) layers were then deposited on a $\mathrm{SiO}_{2} / \mathrm{Si}$ substrate using e-beam evaporation (EXPLORER 14, Denton Vacuum), and were lifted off in acetone to create IDMEs with $2-8 \mu \mathrm{m}$ finger width and spacing. Compared with the conventional one-step lift-off process, this process creates a $\mathrm{SiO}_{2}$ barrier step between the neighbouring electrodes. Such barriers have been shown to provide higher sensitivity compared to coplanar electrodes (Bratov et al. 2008). Previously reported research has revealed that $95 \%$ of the effective electric fields and currents of IDMEs are concentrated above the electrode surface within a distance equivalent to an electrode spacing plus half of an electrode width (Van Gerwen et al. 1998). Thus, in order to maintain high sensitivity in monitoring probe assembly and ELISA, the minimum width and spacing of the IDMEs were designed to be $2 \mu \mathrm{m}$, comparable with their individual lengths. The effective sensing area of the IDME is $2 \mathrm{~mm} \times 2 \mathrm{~mm}$. The impedance sensors were cleaned with 10 minutes of $\mathrm{O}_{2}$ plasma at $150 \mathrm{~W}$ to remove organic residues. A $3 \mathrm{~mm}$-thick Polydimethylsiloxane (PDMS) reaction chamber with a volume of $30 \mu \mathrm{l}$ was fabricated separately and bonded to the sensor substrate. During VLP self-assembly on the impedance sensor, as well as the on-chip ELISA experiments, an additional layer of PDMS is temporarily attached on the PDMS reaction chamber to seal the cavity, therefore, preventing the $30 \mu \mathrm{l}$ liquid sample from rapid evaporation, and maintaining constant analyte concentration and ionic strength. The reaction chamber and cover layer were used throughout the VLP self-assembly on the impedance sensor as well as the on-chip ELISA experiments.

Electrical impedance spectroscopy was performed using an electrochemical workstation (CHI660D, CH Instruments, TX). The excitation used a $50 \mathrm{mV}$ amplitude of alternating current (AC) signal in the frequency range of $10 \mathrm{~Hz}-1 \mathrm{MHz}$ Parallel experiments to monitor the impedance evolution on multiple microsensors were achieved by connecting the sensor electrodes to the CHI660D electrochemical workstation through a multiplexer (CHI684, CH Instruments, TX).

The equivalent electrical circuit of the designed impedance microsensor while monitoring VLP self-assembly and immunoassays is shown in Figure 2. This circuit has been widely used for 
describing the biomedical impedance spectroscopy model in literature (Dak et al. 2014; Hong et al. 2005; Ibrahim et al. 2013). In this model, $R_{c}$ represents the parasitic and contact resistance between the potentiostat and the contact pads of the impedance sensor, $\mathrm{C}_{\text {cell }}$ is the direct capacitive coupling between the IDMEs, $\mathrm{R}_{\mathrm{s}}$ represents the solution resistance between the electrodes, and $\mathrm{C}_{\mathrm{dl}}$ is the double layer capacitance on the electrode/electrolyte interface. During electrical impedance spectroscopy, the conduction current will propagate through $\mathrm{R}_{\mathrm{s}}$ and the displacement current will propagate through $\mathrm{C}_{\text {cell }}$; therefore, the $\mathrm{R}_{\mathrm{s}}$ and $\mathrm{C}_{\text {cell }}$ components are connected in parallel. The solution resistance $\mathrm{R}_{\mathrm{s}}$ is connected in series with the interfacial capacitance $\mathrm{C}_{\mathrm{dl}}$ of the two electrodes. The interfacial capacitance $\mathrm{C}_{\mathrm{dl}}$ contributes primarily to the total impedance of this equivalent circuit when the operating frequency is below $\mathrm{F}_{\text {low }}$, where the total impedance $\mathrm{Z}$, critical cut-off frequency $\mathrm{F}_{\text {low }}$ and interfacial capacitance $\mathrm{C}_{\mathrm{dl}}$ can be expressed as,

$$
\begin{aligned}
& Z \approx R_{s}-j \frac{2}{\omega C_{d l}} \\
& F_{\text {low }} \approx \frac{1}{\pi \cdot R_{s} \cdot C_{d l}} \\
& C_{d l} \approx \frac{\varepsilon_{r} \varepsilon_{0} A}{d}
\end{aligned}
$$

In equation $3, \varepsilon_{\mathrm{r}}$ is the relative dielectric constant of the media directly in contact with the electrode surface and $\mathrm{A}$ is the area of the electrode that is directly in contact with the ionic solution. In the frequency range lower than $F_{\text {low }}$, the total impedance increases with decreasing frequency. The decrease of $\mathrm{C}_{\mathrm{dl}}$ due to dielectric constant $\varepsilon_{\mathrm{r}}$ and effective double layer area changes will also induce the increase in impedance.

\subsection{Sandwiched ELISA on-chip with VLPs as sensing probes}

With cysteine residues genetically expressed on its outer surface, VLP-FLAG sensing probes can be self-assembled onto gold surface through thiol-gold interaction. The self-assembly process on the impedance sensor can be achieved by exposing the gold sensor electrodes to VLP suspension at room temperature. After the VLP-FLAG self-assembled on the impedance sensor surface, on-chip ELISA was performed to validate the VLP probe efficacy in sensing the target anti-FLAG antibodies. Determining factors such as IDME geometries and VLP concentrations used in sensor functionalization were studied in order to achieve better sensing performance in the ELISA process.

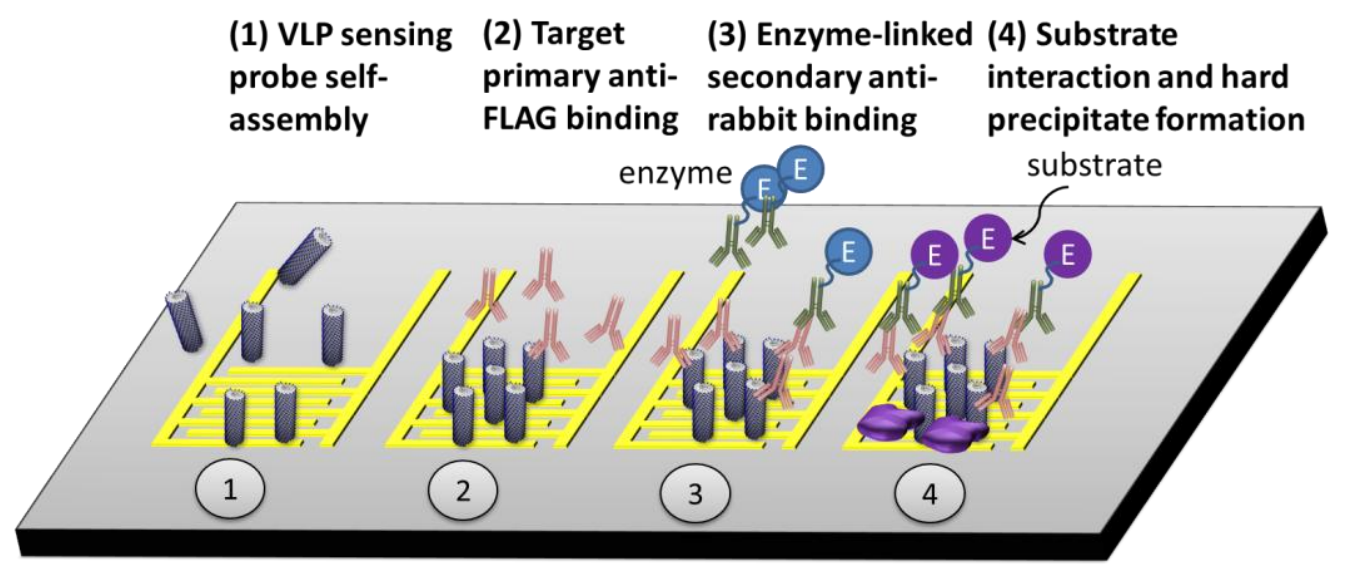

Figure 3: Sandwiched on-chip ELISA using VLP-FLAG 
Sandwiched ELISA (Figure 3) was used as a model system to investigate the sensing efficacy of assembled VLP-FLAG sensing probes to anti-FLAG antibody targets. In the process, VLP-FLAG sensing probes were introduced in the reaction chamber and self-assembled on the impedance sensor surface. After washing off excessive VLPs on the device using buffer solution, a Tris-buffered saline (TBS) (1X) solution containing $12 \mathrm{ng} / \mathrm{ml}-1.2 \mu \mathrm{g} / \mathrm{ml}$ of target primary anti-FLAG IgG antibodies, produced from rabbit (dilution of F7425, Sigma-Aldrich), was introduced in the reaction chamber. A 1:10000 dilution of Goat anti-Rabbit IgG (whole molecule)-Alkaline Phosphatase antibody (A3687, Sigma-Aldrich) was subsequently introduced in the reaction chamber. In the final step, the nitro-blue tetrazolium chloride and 5-bromo-4-chloro-3'-indolyphosphate p-toluidine salt (NBT/BCIP) substrates (Fischer Scientific) were added into the chamber for interacting with the enzyme on the secondary antibody, producing dark-purple insoluble precipitates on the surface. Between all steps, the devices surfaces were washed 3 times using TBS and one time using TBS with $0.05 \%$ Polyoxyethylene (20) sorbitan monolaurate (Tween 20) buffer solutions. The impedance changes were continuously monitored during these experiments.

\section{Results and discussions}

3.1 Microfabricated impedance sensor and its functionalization using VLP self-assembly

Scanning electron microscopy (SEM) is utilized to examine the surface morphology of the impedance sensor before and after VLP self-assembly.

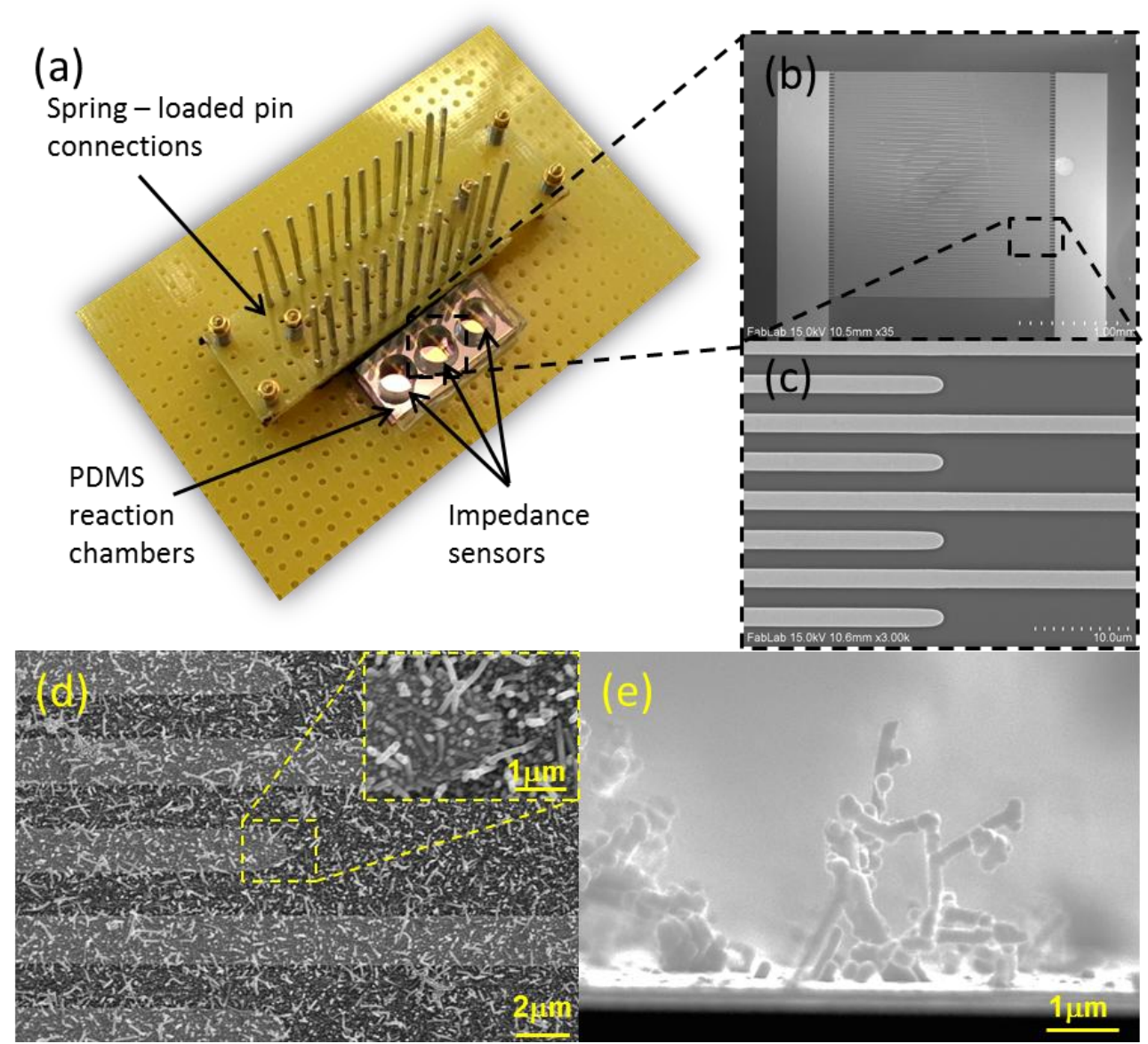

Figure 4. (a) Optical image of the experiment setup for parallel testing of three impedance microsensors; (b) the 4mm ${ }^{2}$ IDME sensing area; (c) the detailed 2 um interdigitated electrode geometries; (d) top-down and (e) cross-sectional views of self-assembled VLP layer on IDMEs 
Figure 4a shows optical images of the fabricated sensor platform and test setup. The sensor platform is composed of three independent sensing units with identical impedance sensor geometries and PDMS micro reaction chambers. The contact pads of the sensors were anchored and electrically connected with the CHI660D potentiostat through spring-loaded probes testing pins. The $\mathrm{Au} / \mathrm{Cr}$ IDMEs achieved the minimum electrode width and spacing of $2 \mu \mathrm{m}$ in the active IDME area of $2 \mathrm{~mm}$ $\times 2 \mathrm{~mm}$ after the lift-off process (Figure $4 \mathrm{~b}$ and $\mathrm{c}$ ).

Figure $4 \mathrm{~d}$ is the SEM image showing the impedance sensor surface after functionalization using 18-hour self-assembly of VLP-FLAG at a concentration of $0.2 \mathrm{mg} / \mathrm{ml}$. Before SEM imaging, the surface was pre-treated with one-hour palladium activation and 4 minutes nickel electroless plating to enhance the contrast in SEM. The nanorod shaped VLPs (white particles in Figure 4d) uniformly cover the impedance sensor surface, forming a functional layer. The cross-sectional SEM image (Figure 4e) shows that the VLPs are quasi-vertically aligned on the electrode surface due to the interaction between the sensor surface and exposed cysteines at the end of each VLP rod. The average length of each VLP is about $1 \mu \mathrm{m}$, and the maximum VLP layer thickness reaches around 3-4 $\mu \mathrm{m}$ due to stacking of VLPs. Impedance sensors with IDME features of $2 \mu \mathrm{m}$ and $8 \mu \mathrm{m}$ (two times the lengths of a single VLP and the maximum VLP layer thickness, respectively) were utilized.

\subsection{Real-time monitoring of VLP self-assembly}

The equivalent circuit model adapted in section 2.2 was utilized to analyze the measured electrical impedance spectrum from $10 \mathrm{~Hz}$ to $1 \mathrm{MHz}$ during VLP self-assembly, aiming at a fundamental understanding of the sensor electrical property changes in this process.
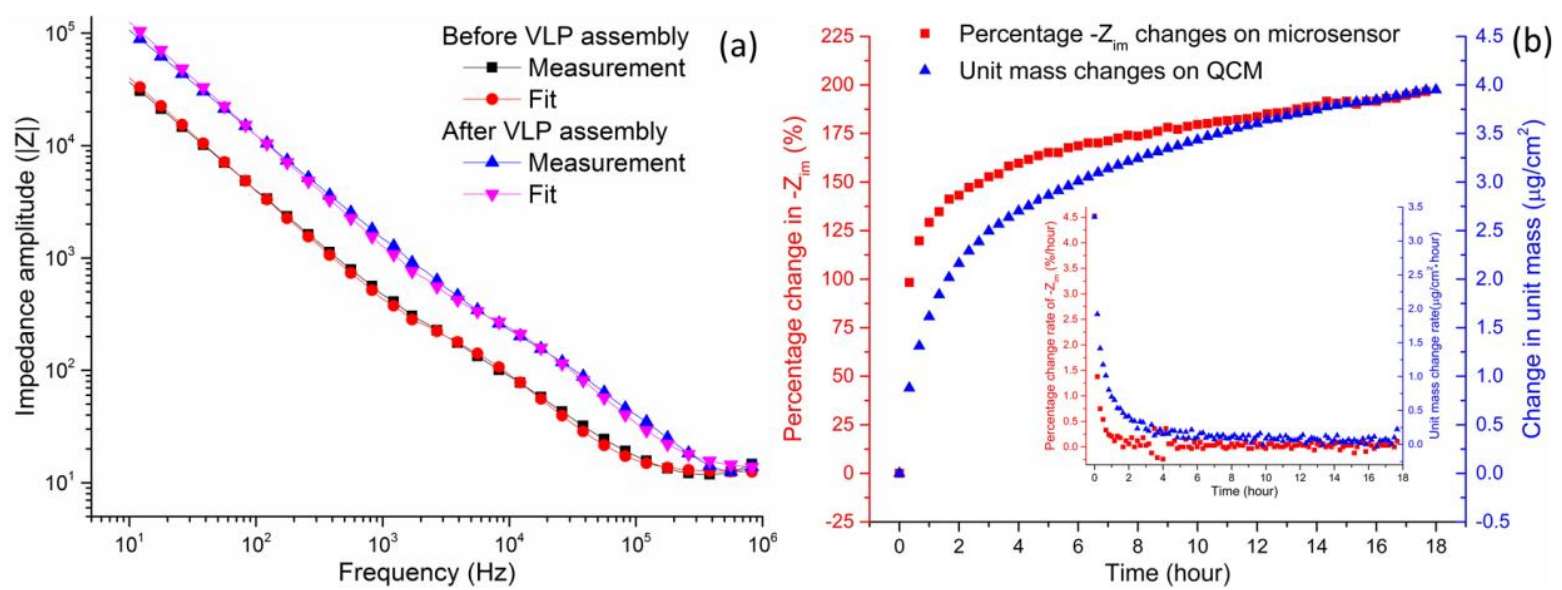

Figure 5. (a) Impedance spectrums from measurement and fitting using the equivalent circuit before and after VLP self-assembly on the impedance microsensor; (b) real-time impedance and mass loading measurement using the developed impedance microsensor and quartz crystal microbalance, respectively

The impedance spectra from both direct measurements and fits using the equivalent circuit model before and after VLP self-assembly are plotted in Figure 5a. The impedance fit from the equivalent circuit matches well with the experimental results in the entire frequency range from $10 \mathrm{~Hz}$ to $1 \mathrm{MHz}$ $\left(\mathrm{R}^{2}=0.999\right.$ for both fits before and after VLP assembly), validating the suitability of implementing this circuit to describe the electrical behaviour of the sensor. After VLP assembly, the amplitude of the electrical impedance from $10 \mathrm{~Hz}$ to $1 \mathrm{kHz}$ showed a significant approximately 3-fold increase compared to that measured before VLP assembly. The extracted electrical parameters from fitting the measured spectra using the circuit model are listed in Table S1 (see ESI). In the presence of VLPs on the electrode surface, the dielectric capacitance $\mathrm{C}_{\text {cell }}$ decreased by $69 \%$ compared to bare electrodes due to the lower effective dielectric constant $\left(\varepsilon_{\mathrm{r} \text {,water }} \approx 80, \varepsilon_{\mathrm{r} \text {,protein }} \approx 4-13\right)$ after the VLP attachment. 
The interfacial capacitance $\mathrm{C}_{\mathrm{dl}}$ is the dominant capacitive component compared with $\mathrm{C}_{\text {cell }}$. It showed about $68 \%$ decrease during the VLP self-assembly process which indicate the VLP surface coverage. The VLP assembly also decreases the effective electrode area on the current conduction path, thus increasing the observed solution resistance. The higher impedance amplitude in the frequency of 10 $\mathrm{Hz}-1 \mathrm{MHz}$ after the VLP assembly is due to the combined effect of higher solution resistance and lower capacitance. From these electrical components, the low cut-off frequency $F_{\text {low }}$ of this microsensor is $2 \mathrm{kHz}$. Therefore, using the frequency range below $2 \mathrm{kHz}$, the interfacial capacitance $\mathrm{C}_{\mathrm{dl}}$ is the determining component for impedance, and the imaginary part of the impedance is dominant compared to the real part. At the frequency of $100 \mathrm{~Hz}$, the model fit also shows highest accuracy with an error as low as $0.16 \%$. In the later study, the imaginary part of the impedance at the frequency of $100 \mathrm{~Hz}$ will be used for analyze the real-time VLP assembly and ELISA process.

The imaginary part of the complex electrical impedance on a sensor with $8 \mu \mathrm{m}$ electrode feature size, about 2 times the VLP layer thickness observed, was utilized to analyze the VLP self-assembly dynamics over an 18 hours duration (Figure $5 b$ ). The response of the impedance microsensors is also compared to a $5 \mathrm{MHz}$ quartz crystal microbalance (QCM) sensor (INFICON, Switzerland) to validate the efficacy of the developed device. QCM platforms have previously been shown as reliable systems for biosensing such as DNA hybridization, protein and cell attachment detections (Hianik et al. 2005; Mannelli et al. 2003; Rodahl et al. 1997). However, due to the need for bulk quartz crystal for generating resonance, the miniaturization of QCM to fit in portable sensing systems is very challenging. Figure $5 \mathrm{~b}$ shows the comparison of impedance (red squares) and unit mass loading (blue triangles) detected by the designed impedance microsensor and the QCM, respectively. Both sensor systems responded similarly to the VLP self-assembly, where the impedance amplitude (red squares) and mass loading (blue triangles) trends reflected the rapid attachment of VLP sensing probes to the surfaces in the initial 3 hours followed by a gradual increase of attachment throughout the 18 hours of self-assembly. The inset in Figure 5b shows the impedance and mass changing rates in the impedance sensor and QCM systems, respectively. The mass-loading rate on the QCM decreased from the initial $3.4 \mu \mathrm{g} / \mathrm{cm}^{2}$.hour to $1 \mu \mathrm{g} / \mathrm{cm}^{2}$.hour (a $71 \%$ decrease) in the first hour of VLP assembly. In the same time scale, the response of the impedance sensor degrades more dramatically from $4.5 \%$ /hour to $0.25 \%$ /hour (a 94\% decrease). The accumulation of VLPs that beyond the electric field and current distributions near the impedance sensor electrode surfaces may attribute to the relatively quick loss of response to VLP assembly using the impedance sensor.

These results show that the impedimetric sensors are capable of real-time monitoring of VLP sensing probe self-assembly on the transducer surfaces of a more compact platform than the traditional QCM. The impedance change reveals that the first 3 hours during VLP self-assembly is the most critical period to saturate a sensor surface.

\subsection{Impedimetric on-chip ELISA}

The full ELISA procedure is performed on the VLP functionalized impedance sensors to study the impact of VLP concentrations and sensor geometries to the sensing performance. Moreover, the sensor response to increasing concentrations of target antibody in the full ELISA was characterized. 

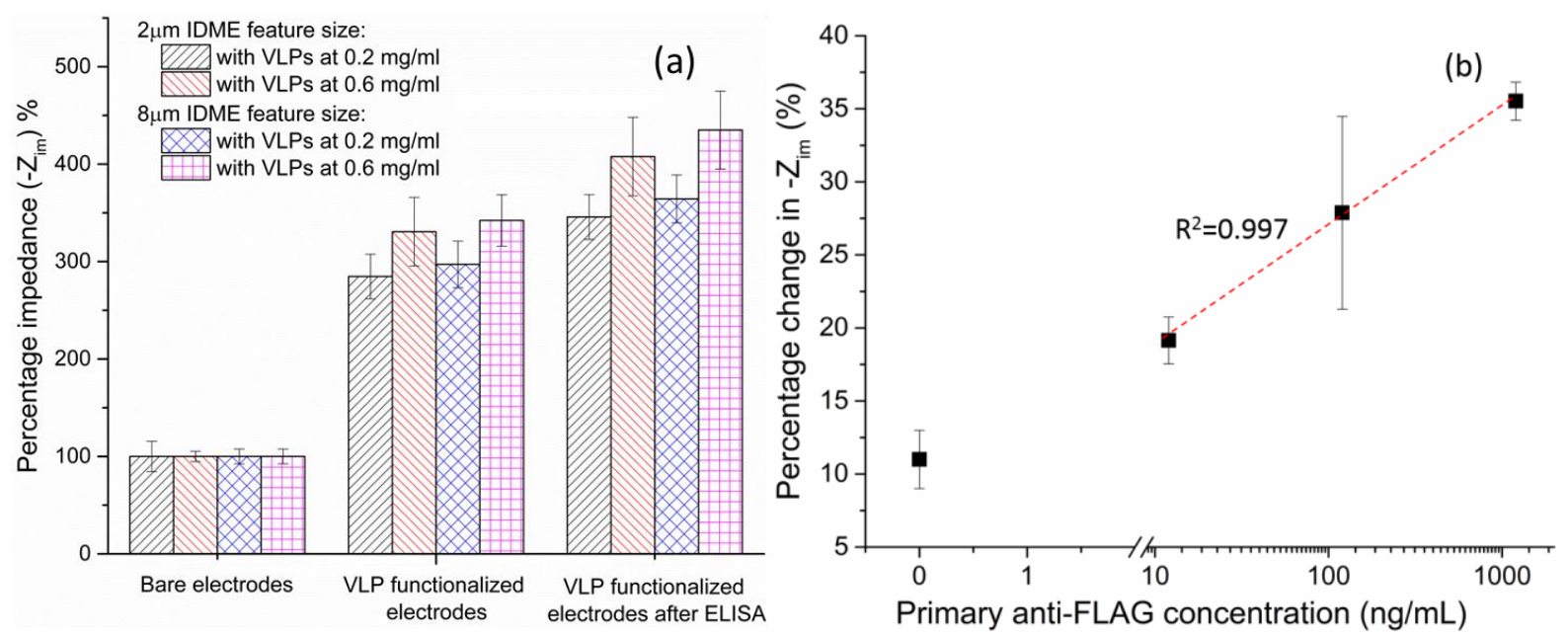

Figure 6. (a) The percentage impedance $-Z_{\text {im }}$ during VLP self-assembly and ELISA process obtained by impedance microsensor; (b) percentage impedance (imaginary part) after ELISA process with different target primary antibody concentrations. The error represents the standard deviation of the mean $(n=3)$

Figure 6a shows the changes of the imaginary part of the electrical impedance observed using different IDMEs functionalized by VLPs at different concentrations. The impedance increases significantly during the VLP assembly process due to both the decrease in direct electrode/electrolyte interfacial area and the lower effective dielectric constant, as discussed previously. The slight decreases in the impedance during both antibody binding steps may be attributed to the disassociation of the non-specifically bound VLPs on the sensor surface (through bonds other than thiol-gold), and diffusion into the Tris-buffer during the testing. The sensor responses in the full ELISA also showed that the largest overall impedance shifts are from experiments using the IDMEs with $8 \mathrm{~mm}$ feature size and $0.6 \mathrm{mg} / \mathrm{ml}$ concentration VLPs.

In order to understand the determinant factor among VLP concentrations and electrode geometries, two-way analysis of variance (ANOVA) is utilized to analyze the statistical significance of the data sets in Figure 6a. The ANOVA test in Table S2 (see ESI) shows that VLP concentration is the only major source of variation $\left(\mathrm{F}=15.72>\mathrm{F}_{\text {crit }}, \mathrm{p}=0.0041<0.05\right)$ that controls the percentage impedance level after ELISA process. The ANOVA test also shows that, regardless of electrode feature size, all impedance sensors response similarly as the VLP concentration increases. These analytical results indicate that VLP concentration is the dominant factor for the VLP-based impedance biosensor performance. A higher VLP concentration will contribute to enhanced impedance sensor responses in the immunoassay.

Table S3 summarizes the percentage relative imaginary impedance changes in the VLP assembly and the ELISA processes. In VLP assembly, the relative changes were compared between the imaginary impedance amplitudes measured with VLP functionalized IDMEs and bare IDMEs. In the ELISA, the relative changes in impedance were compared between the measured imaginary impedance after precipitate formation (step 4 described in section 2.3) and after VLP self-assembly (step 1 described in section 2.3). Both impedance sensor designs show high sensitivity to VLP attachment on the sensor surface, measuring up to about $240 \%$ increase in imaginary impedance using $8 \mu \mathrm{m}$ IDMEs and $0.6 \mathrm{mg} / \mathrm{ml}$ VLP concentration. However, compared with the $8 \mu \mathrm{m}$ feature size impedance sensors, the impedance sensors with finer electrode features of $2 \mu \mathrm{m}$ do not show higher impedance shifts during the ELISA process, and it showed less VLP-FLAG concentration dependency. This may be attributed to the saturation of the effective sensing area by the dense VLP layer that degrades the sensor performance during the immunoassay. 
Figure $6 \mathrm{~b}$ shows the total percentage of imaginary impedance amplitude change in the ELISA process when different concentrations of anti-FLAG antibody were used. Impedance sensors with 8 $\mu \mathrm{m}$ electrode width and spacing functionalized by $0.6 \mathrm{mg} / \mathrm{ml}$ VLP-FLAG sensing probes were used in this study. Tris-buffer solution with no anti-FLAG was introduced as the control in the ELISA. In the control experiment, the impedance change was $11 \%$ due to non-specific binding of secondary antibody and precipitate formation on the sensor. In the sensing experiments, the impedance sensors detected $19 \%, 28 \%$ and $36 \%$ of total impedance increases after the entire ELISA procedure when 12 $\mathrm{ng} / \mathrm{ml}, 120 \mathrm{ng} / \mathrm{ml}$ and $1.2 \mu \mathrm{g} / \mathrm{ml}$ of target anti-FLAG primary antibody were used, respectively. The error bars in Figure $6 \mathrm{~b}$ represent the standard deviation of the results from three independent experiments using newly functionalized sensors at identical conditions. Based on the linear correlations $\left(\mathrm{R}^{2}=0.997\right)$ between the percentage impedance change and antibody concentration (log scale), the sensitivity of the VLP functionalized microsensor can be calculated as $8.7 \%$ of the total impedance per magnitude of antibody concentration $(\mathrm{ng} / \mathrm{ml})$. The detection limit of target antibody for the on-chip impedimetric ELISA is $9.1 \mathrm{ng} / \mathrm{ml}$.

\section{Summary}

Impedance microsensors were developed and tailored toward comprehensive studies of the viruslike particle nanosensing probe self-assembly dynamics on sensor surfaces and impedimetric responses during immunoassays. The genetic modifications of the TMV coat proteins enable the autonomous formation of VLP nanosensing probes carrying cysteines and affinity peptides in bacteria cells. VLP sensing probes quasi-vertically self-assembled on the microsensor surface and form a functional layer without the need for chemical treatment of the surface. Continuous impedance monitoring over 18 hours of VLP-FLAG assembly showed the rapid attachment of VLP on the impedance sensor surface in 3 hours. With the similar sensing results from the impedance sensor and QCM, the impedimetric microsensors proved to be promising for the real-time monitoring of macromolecular sensing probe assembly in a more compact system. The on-chip ELISA experiments validated the sensing efficacy of the VLP-functionalized impedance sensors in detecting the presence of down to $9.1 \mathrm{ng} / \mathrm{ml}$ target antibodies.

This work is the first demonstration of real-time monitoring of high-surface area TMV-VLP macromolecular sensing probe self-assembly dynamics with an impedimetric microsensor. This experimental study provided important self-assembly parameters of the VLP sensing probes to identify the effective VLP self-assembly time and its surface coverage on sensors. The VLP sensing probes can potentially be used as programmable multi-target sensing receptors by altering the peptide sequence on the VLP protein surface. The probes can also be used to immobilize large quantity of antibodies on a transducer surface. In summary, this work will contribute to the future development of programmable VLP-based biosensors with autonomous sensor functionalization and enhanced sensing performances.

\section{Acknowledgements}

The work was supported by the Army Research Office Biochemistry Program (Grant No. W911NF-14-1-0286) and National Science Foundation Nanomanufacturing Program (Grant No. NSF-CMMI 0927693). The authors would like to acknowledge the staff in Maryland Nanocenter for the support in fabrication process.

\section{REFERENCES}

Ayliffe, H.E., Bruno Frazier, A., Rabbitt, R., 1999. J. Microelectromech. Syst. 8(1), 50-57.

Bratov, A., Ramon-Azcon, J., Abramova, N., Merlos, A., Adrian, J., Sanchez-Baeza, F., Marco, M.P., Dominguez, C., 2008. Biosens. Bioelectron. 24(4), 729-735. 
Brown, A.D., Naves, L., Wang, X., Ghodssi, R., Culver, J.N., 2013. Biomacromolecules 14(9), 31233129.

Culver, J.N., Brown, A.D., Zang, F., Gnerlich, M., Gerasopoulos, K., Ghodssi, R., 2015. Virology 479, 200-212.

Dak, P., Ebrahimi, A., Alam, M.A., 2014. Lab Chip 14(14), 2469-2479.

Fan, X., Naves, L., Siwak, N., Brown, A., Culver, J., Ghodssi, R., 2015. Nanotechnology 26(20), 205501.

Fan, X, Gerasopoulos, K., Brown, A., Siwak, N., Culver, J., Ghodssi, R., 2010. Sensors, 2010 IEEE, 2629-2633.

Fan, X, Pomerantseva, E., Gnerlich, M., Brown, A., Gerasopoulos, K., McCarthy, M., Culver, J., Ghodssi, R., 2013. J. Vac. Sci. Technol., A 31(5).

Gerasopoulos, K., Pomerantseva, E., McCarthy, M., Brown, A., Wang, C., Culver, J., Ghodssi, R., 2012. ACS Nano 6(7), 6422-6432.

Hianik, T., Ostatná, V., Zajacová, Z., Stoikova, E., Evtugyn, G., 2005. Bioorg. Med. Chem. Lett. 15(2), 291-295.

Hong, J., Yoon, D.S., Kim, S.K., Kim, T.S., Kim, S., Pak, E.Y., No, K., 2005. Lab Chip 5(3), 270279.

Ibrahim, M., Claudel, J., Kourtiche, D., Nadi, M., 2013. J. Electr. Bioimp. 4(1), 13-22.

Katz, E., Willner, I., 2003. Electroanalysis 15(11), 913-947.

Mandal, P., Biswas, A., Choi, K., Pal, U., 2011. Am. J. Food Technol. 6(2), 87-102.

Mannelli, I., Minunni, M., Tombelli, S., Mascini, M., 2003. Biosens. Bioelectron. 18(2), 129-140.

Moreno-Hagelsieb, L., Lobert, P., Pampin, R., Bourgeois, D., Remacle, J., Flandre, D., 2004. Sens. Actuators, B 98(2), 269-274.

Narsaiah, K., Jha, S.N., Bhardwaj, R., Sharma, R., Kumar, R., 2012. J. Food Sci. Technol. 49(4), 383406.

Roda, A., Guardigli, M., 2012. Anal. Bioanal. Chem. 402(1), 69-76.

Roda, A., Pasini, P., Mirasoli, M., Michelini, E., Guardigli, M., 2004. Trends Biotechnol. 22(6), 295303.

Rodahl, M., Höök, F., Fredriksson, C., Keller, C.A., Krozer, A., Brzezinski, P., Voinova, M., Kasemo, B., 1997. Faraday Discuss. 107, 229-246.

Van Gerwen, P., Laureyn, W., Laureys, W., Huyberechts, G., De Beeck, M.O., Baert, K., Suls, J., Sansen, W., Jacobs, P., Hermans, L., Mertens, R., 1998. Sens. Actuators, B 49(1-2), 73-80.

Varshney, M., Li, Y.B., Srinivasan, B., Tung, S., 2007. Sens. Actuators, B 128(1), 99-107.

Yang, L., Bashir, R., 2008. Biotechnol. Adv. 26(2), 135-150.

Zang, F., Fan, X.Z., Gerasopoulos, K.D., Ben-Yoav, H., Brown, A.D., Culver, J.N., Ghodssi, R., 2013. SENSORS, 2013 IEEE, 1-4.

Zang, F., Gerasopoulos, K., Fan, X.Z., Brown, A.D., Culver, J.N., Ghodssi, R., 2014. Chem. Commun. 50(85), 12977-12980.

Zou, Z., Kai, J., Rust, M.J., Han, J., Ahn, C.H., 2007. Sens. Actuators, A 136(2), 518-526. 